\title{
The application of ARIMA-RBF model in urban rail traffic volume forecast
}

\author{
He-Jiuran \\ School of Traffic and Transportation, Beijing Jiaotong \\ University \\ Beijing, China \\ 11120860@bjtu.edu.cn
}

\author{
Si-Bingfeng \\ School of Traffic and Transportation, Beijing Jiaotong \\ University \\ Beijing, China \\ bfsi@bjtu.edu.cn
}

\begin{abstract}
Due to various factors, passenger flow has nonlinear characteristics. Autoregressive Integrated Moving Average Model (ARIMA model) is suitable for non-stationary time series forecasting while RBF neural network is a kind of forward neural network which has good approximation performance and is suitable for processing nonlinear problem. In this paper, we combine the ARIMA model and RBF neural network model to formulate the ARIMA - RBF model by analyzing passenger flow' $s$ temporal characteristics, the mechanism of ARIMA model with RBF model. We use the proposed model which used to forecast Beijing urban rail transit passenger flow and obtain a good prediction result.
\end{abstract}

Keywords- Railway traffic; Passenger flow forecast; Combination Forecasting; RBF neural network; ARIMA model

\section{INTRODUCTION}

Passenger flow prediction is the base of urban rail transit planning and operation management, which has become an important part in urban rail transit construction. Time series method is the most widely used passenger flow prediction method. The time series method originated in 1927. In recent years, ARIMA model has been one of the main methods of prediction. It is the basic thought's foundation of many kinds of time series methods. Yao et al ${ }^{[1]}$ applied ARIMA model in traffic flow trend forecast, and got a better prediction results. Chi et al ${ }^{[2]}$ predicted the coal consumption with ARIMA model. ARIMA method now has complete modeling methods while it still has some limitations. It assumes that the analysis of time series is linear, so nonlinear time series prediction can't achieve the best results.

Neural network is a kind of new prediction methods, and it is suitable for complex, nonlinear conditions. It doesn't need to establish accurate mathematical model, and has good self-organizing, adaptability, strong learning ability and antiinterference ability ${ }^{[3]}$.The related models include BP neural network model, multi-layer perceptron neural network and radial basis function neural network, dynamic neural network, the neural network model, and so on. These models have been widely applied in the field of prediction. For example, Deng ${ }^{[4]}$ used the BP neural network model to predict highway passenger quantity, and has obtained a better prediction results. Xiao et al ${ }^{[5]}$ used RBF neural network to forecast highway short-term traffic flow and the results showed that the RBF neural network could accurately predict high-speed traffic flow. Li Xiaojun ${ }^{[6]}$ proposed to use radial basis neural network to predict short-term railway passenger flow.

In recent years, many scholars consider to use different prediction methods to build combinatorial prediction model so as to improve the accuracy of prediction. Tsung-Hsien Tsai ${ }^{[7]}$ proposed sequence unit neural network model and parallel combination neural network to forecast the railway passenger transport demand. Zheng ${ }^{[8]}$ combined Bayesian method with neural network for the short-term prediction of highway traffic flow, and the results showed that the combinatorial model predicts are more effective than single neural network model.

Urban rail transit passenger flow has sequence characteristics. It has year, month, week, day changes rules. One of the most obvious sequence characteristics of passenger flow is week cycle. In each cycle, passenger flow has similar changing trends. Due to a variety of factors, passenger flow's change in each cycle is different and has nonlinear characteristics. In this paper, we build ARIMARBF model to forecast the urban rail transit passenger flow which has periodic and nonlinear characteristics. The main job of this paper are: (1) to sum up the passenger flow sequence characteristics from the passenger flow line chart and select the history data to predict the passenger flow. (2) using ARIMA model to forecast the passenger flow and the passenger flow prediction result contains linear characteristic, the nonlinear characteristic is included in the ARIMA prediction error.(3) using RBF neural network model to forecast the ARIMA prediction error and establishing ARIMA-RBF model.

\section{ARIMA MODEL}

\section{A. ARIMA MODEL}

The actual passenger flow sequence is always a nonstationary time series, and it is needed to transform the nonstationary time series into stationary time series. The conversion method is difference method. Supposing $x_{t}$ is a nonstationary time series.

$$
y_{t}=(1-B)^{d} x_{t}
$$

Where $y_{t}$ and $\mathrm{B}$ are stationary time series and difference operator.

If the time series is cycle time series and the cycle is $S$, 


$$
y_{t}=\nabla_{s} x_{t}=\left(1-B^{s}\right) x_{t}
$$

Then in $\operatorname{ARMA}(\mathrm{p}, \mathrm{q})$ model, the time series $y_{t}$ has the form

$$
\begin{aligned}
y_{t}= & \theta_{0}+\phi_{1} y_{t-1}+\phi_{2} y_{t-2}+\cdots+\phi_{p} y_{t-p} \\
& +\varepsilon_{t}-\theta_{1} \varepsilon_{t-1}-\theta_{2} \varepsilon_{t-2}-\cdots-\theta_{q} \varepsilon_{t-q}
\end{aligned}
$$

Where $y_{t}$ is the observation value at $t$ time. $\left\{\varepsilon_{t}\right\}$ is white noise sequence with mean of zero. $\phi_{i}(i=1,2,3 \ldots p)$ and $\theta_{j}(i=1,2,3 . . q)$ are model parameters. $\mathrm{P}$ and $\mathrm{q}$ are integers and often referred to as orders of the model. If $q=0$, then Eq. (3) becomes an AR model of order $p$. When $p=0$, the model reduces to an MA model of order q. One central task of the ARIMA model building is to determine the appropriate model order $(p, q)$.

By applying operator polynomial, the Eq.(3) can be expressed as

$$
\phi(B) y_{t}=\theta(B) \varepsilon_{t}
$$

$$
\begin{aligned}
& \text { where, } \phi(B)=1-\phi_{1} B-\phi_{2} B^{2}-\cdots-\phi_{p} B^{p} \\
& \qquad \theta(B)=1+\theta_{1} B+\theta_{2} B^{2}+\cdots+\theta_{q} B^{q}
\end{aligned}
$$

$\operatorname{ARIMA}(d, p, q)$ model has the form

$$
\phi(B)(1-B)^{d} x_{t}=\theta(B) \varepsilon_{t}
$$

\section{B. The modeling process of ARIMA model}

ARIMA model modeling process can be divided into four steps.

First of all, judge the passenger flow sequence's stationarity. If passenger flow sequence is not stationary series, we need to do difference calculation or logarithmic difference and transform the original passenger flow sequence into stationary sequence.

Second, calculate autocorrelation coefficient and partial autocorrelation coefficient, and determine the number of $\mathrm{p}$ and q. In this article, we use AIC criterion to determine the $\mathrm{p}$ and $\mathrm{q}$.

Third, estimate the unknown parameters and test parameters.

Finally, diagnosis analyzing. It is need to confirm the rationality of the model.

\section{RBF NEURAL NETWORK MODEL}

RBF neural network is a kind of forward neural network with good approximation performance and has the advantages of simple structure, fast learning, no local minimum and so on ${ }^{[3]}$. RBF neural network has three layer structures: input layer, hidden layer and output layer. The general RBF neural network structure is shown in figure 1 .

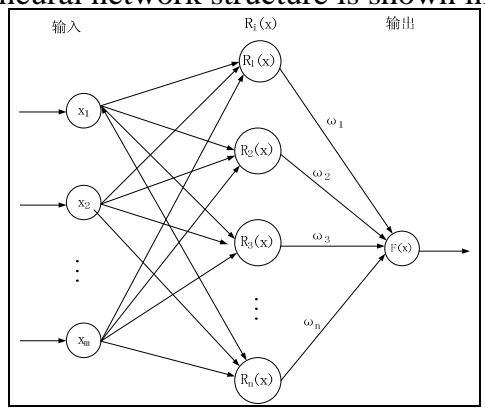

Figure 1. A general architecture of RBF neural network

Where $x_{1}, x_{2}, \ldots, x_{m}$ are input values, $n$ is the hidden node, $F(x)$ denote the output of the model, $\left(\omega_{1}, \omega_{2}, \ldots, \omega_{n}\right)$ represent the weight between Hidden layer and output layer. The weight between input layer and Hidden layer are 1.

The output of hidden layer nodes $i$ is

$$
u_{i}=R\left[\frac{\left\|X-C_{i}\right\|}{\sigma_{i}}\right]
$$

Where $i=1,2, \cdots, m, \mathrm{R}$ is radial basis function, $C_{i}$ is the center of radial basis function's neuron $i, \sigma_{i}$ is the width parameters of the radial basis function neurons, $\left\|X-C_{i}\right\|$ is European norm.

Hidden layer can choose different function. It is usually to use Gaussian function as the radial basis function, namely

$$
R(x)=\exp \left(-\frac{x^{2}}{2}\right)
$$

So the output of RBF neural network is

$$
F(x)=\sum_{i=0}^{m} w_{i} \exp \left(-\frac{\left\|X-C_{i}\right\|^{2}}{2 \sigma_{i}^{2}}\right)
$$




\section{ARIMA-RBF MODEL}

ARIMA model is suitable for linear condition forecasting while RBF neural network model is suitable for nonlinear condition forecasting. In fact, passenger flow's change not only has linear rule but also has nonlinear law. In this article, we combine ARIMA model with RBF neural network model and establish ARIMA-RBF model. ARIMARBF model can both fit passenger flow sequence's linear rules and nonlinear law.

It may be reasonable to consider a passenger flow series to be composed of a linear autocorrelation structure and a nonlinear component. That is,

$$
y_{t}=L_{t}+N_{t}
$$

Where $L_{t}$ denotes the linear component and $N_{t}$ denotes the nonlinear component. These two components have to be estimated from the data. First, we use ARIMA to model the linear component, then the nonlinear relationship is contained in the residuals from the linear model. Let $e_{t}$ denote the residual at time $t$ from the linear model, then

$$
e_{t}=y_{t}-\hat{L}_{t}
$$

Where $\hat{L}_{t}$ is the predicted value of ARIMA model at time $t$. $e_{t}$ contains nonlinear relationship of the passenger flow. By modeling residuals using RBF model, nonlinear relationships can be discovered. With $n$ input nodes, the RBF model for the residuals will be

$$
e_{t}=f\left(e_{t-1}, e_{t-2}, \cdots, e_{t-n}\right)+\varepsilon_{t}
$$

Where $f$ is a nonlinear function determined by the RBF neural network and $\varepsilon_{t}$ is the random error. Let RBF neural network prediction error results is $\hat{N}$, then

$$
\hat{y}_{t}=\hat{L}_{t}+\hat{N}_{t}
$$

The analysis shows that ARIMA - RBF combination forecasting model is divided into two parts. First, the passenger sequence linear prediction with ARIMA model. Then we use RBF neural network to forecast ARIMA prediction error. The hybrid model combines the unique feature and strength of ARIMA model and ANN model in determining different patterns. Thus, it could be advantageous to improve the overall modeling and forecasting performance.

\section{EMPIRICAL RESULTS}

\section{A. Beijing rail transit passenger flow sequence} characteristics analysis

In this article, we select the Beijing metro line one's passenger flow from February 21, 2011 to on May 27, 2012 to forecast passenger flow from May 28, 2012 to June 3.The passenger volume change trend chart is shown as below.

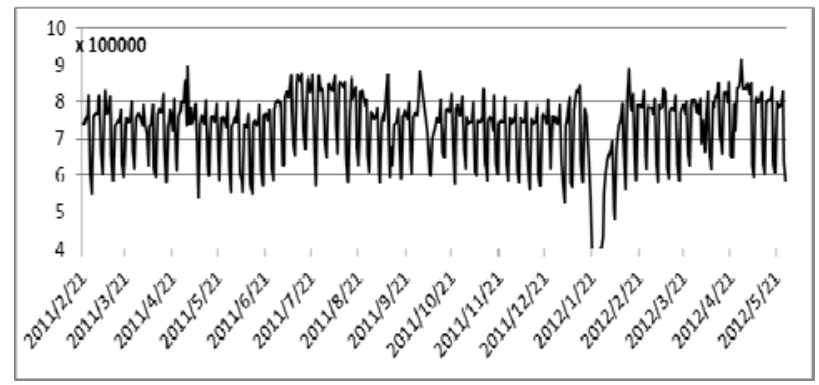

Figure 2. The change trend of line one passenger flow during February 21, 2011 to May 27, 2012

From the Fig.2 we can see that passenger flow volume in every week has similar change trend. The difference between holidays and ordinary day is bigger. So we choose full week ordinary day passenger flow from February 21, 2011-2012 to May 27 as history passenger data. The statistics time interval is one day.

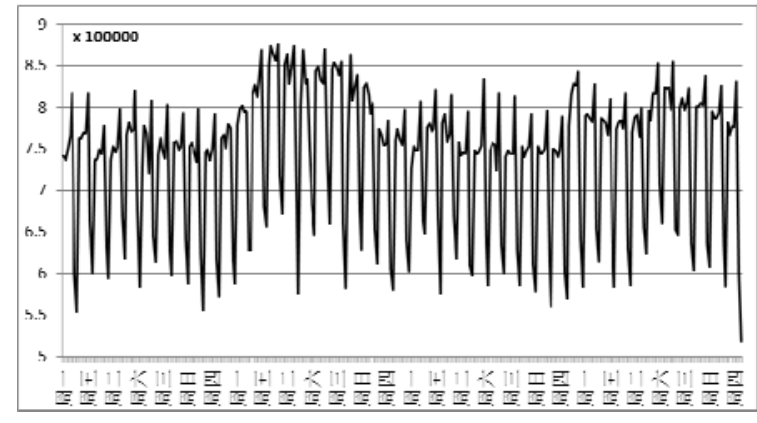

Figure 3. The change trend of line one ordinary day passenger flow during February 21, 2011 to May 27, 2012

As we can see, between February 21, 2011 and May 27, 2012, line one's ordinary day passenger flow occur regular changes within a week. Monday to Thursday are working days, passenger volume's change is not very big. Friday's passenger volume is significantly big. On Saturday and Sunday, the passenger flow number declines, and is far less than the working days. In addition, we can see ordinary day passenger flow presents the volatility. It is stationary time series. So the passenger flow sequence has nonstationarity and cyclical characteristics.

\section{B. Prediction Results}

In this paper, we use full weeks' ordinary day passenger flow during February 21, 2011 to May 27, 2012 to forecast the passenger flow during May 28, 2012 to June 3. First of 
all, we transform the non-stationary passenger flow sequence into stationary passenger flow sequence by difference calculating. Then, we use ARIMA model and the passenger flow data during February 21, 2011 to December 18, 2011 to predict passenger flow during January 9, 2012 May 27, 2012 and get ARIMA model prediction error. The prediction error can be used as the input of RBF neural network, and we get the prediction value of ARIMA model error. At last, adding the ARIMA model prediction value and RBF model's prediction value, we can get the final passenger flow prediction during May 28, 2012 - July 3, 2012.

In this paper, we use ARIMA model, RBF neural network model and ARIMA - RBF model to predict Beijing metro line one's passenger flow during May 28, 2012 to June 3, 2012 and use mean absolute relative percentage error ( $M A P E$ ) as the prediction results' evaluation indicator. The calculation formula is:

$$
M A P E=\left|\frac{\left(y_{i}-\hat{y_{i}}\right)}{y_{i}}\right| \times 100,(i=1,2,3 \cdots, 7)
$$

Where $y_{i}$ and $\hat{y}_{i}$ are the predicted value and the observation value on day $i$ in the forecast week. The MAPE of three models in the forecast week is shown as follow.

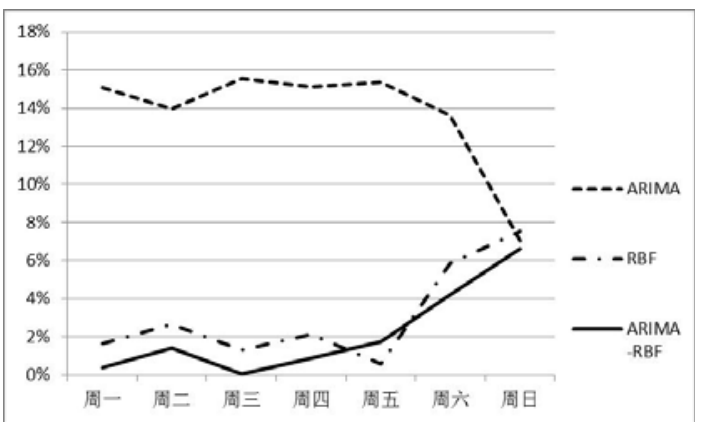

Figure 4. The mean relative absolute percentage error of three models.

In the fig.4, we can conclude that, the $M A P E$ of ARIMA - RBF model is the minimum. ARIMA model's $M A P E$ is the largest. ARIMA - RBF model's average daily forecast error is $2 \%$ and ARIMA model's average prediction error is $14 \%$. RBF neural network's prediction error is between the ARIMA model and ARIMA - RBF model. So ARIMA-RBF model's predict result is the best.

\section{CONCLUSION}

In this paper, we propose to take a combining approach for urban rail transit passenger flow forecasting. The linear ARIMA model and the nonlinear RBF model are used jointly, aiming to capture different forms of relationship in the passenger flow series data. The hybrid model takes advantage of ARIMA model and RBF model in linear and nonlinear modeling.

For real problems that have linear and nonlinear correlation structures, the ARIMA-RBF model can be an effective way to improve forecasting performance. The example's result with real passenger flow data clearly suggest that the hybrid model performs better than each single model used in the hybrid model.

\section{ACKNOWLEDGMENT}

We are grateful to Professor Si and anonymous referees for detailed comments and suggestions which have helped improve the paper substantially. The work described in this article is supported mainly by grants from the National Natural Science Foundation of China (Project No. 71071016) and the Key Technology Research and Development Program of the Ministry of Science and Technology of China (Project Nos. 2011BAG01B01-1).

\section{REFERENCES}

[1] Yao Yafu,Cao Feng. Sneddon,Short-time Traffic Flow Prediction based on ARIMA [J],Technology \& Economy in Areas of Communications,2006,(3):105-107.

[2] Chi Qishui,Liu Xiaoxue. Study on the Application of ARIMA Model in Forecasting China's coal Consumption [J], Energy Research and Information,2007,23(2):117-123.

[3] Gao Ning,Zhang Jianzhong. Application of RBF Neural Network Model based OR MATLAB [J], The Agricultural Network Information,2009,2: 110-116.

[4] Wu Deng,Wen Li,Xin hua Yang. A Novel Hybrid Optimization Algorithm of Computational Intelligence Techniques for Highway Passenger Volume Prediction[J]. Expert Systems with Applications, 2011, 38: 4198-4205.

[5] Jian Mei Xiao,Xi Huai Wang. Study on Traffic Flow Prediction Using RBF Neural Network. Proceedings of the Third International Conference on Machine Learning and Cybernetics, Shanghai, 2004: 26-29.

[6] LI Xiaojun, LV Xiaoyan, LIU Jun. Forecast of Railway Short-term Passenger Flow based on RBF Neural Network[J]. Railway Transport and Economy, 2011, 33(6):86-89.K. Elissa, "Title of paper if known," unpublished.

[7] Tsung-Hsien Tsai, Chi-Kang Lee, Chien-Hung Wei. Neural Network based Temporal Feature Models for Short-term Railway Passenger Demand Forecasting [J]. Expert Systems with Applications, 2009, 36: 3728-3736.

[8] Wei Zhongzheng,Der Horng Lee,Qi Xin Shi. Short-term Freeway Traffic Flow prediction: Bayesian combined Neural Network Approach [J]. Journal of Transportation Engineering, 2011, 132(2): 114-121.

[9] G. Peter Zhang. Time Series Forecasting using a Hybrid ARIMA and Neural Network Model [J]. Neurocomputing, 2003, 50: 159-175.

[10] Zhang Jigang,Liang Na, Combinational Forecasting Model Based on ARIMA and ANN [J], Journal of China Three Gorges University,2008,30(4):86-88. 